MODELING, IDENTIFICATION AND CONTROL, 2003, VOL. 24, No. 4, 193-203

doi:10.4173/mic.2003.4.1

\title{
Control of the Amplitude in a Surging Balling Drum Circuit, a New Approach to an Old Problem
}

\author{
KNUT RAPP* and PER-OLE NYMAN† \\ Keywords: Balling drums, surging, estimation, extended Kalman filter, \\ thresholding
}

In this paper we suggest a new method for controlling the balling drums used in the iron ore industry. We suggest that a cluster of drums are controlled collectively rather than individually. Further, we investigate the possibility of using an extended Kalman filter for estimating the amplitude and frequency of the oscillations in such drums. The filters thresholding point is identified, and the area for which the filter is usable is given. Finally a simulation of the proposed control scheme, based on a simple nonlinear model, is presented.

\section{Introduction}

Use of balling drums has become common in many parts of the industry. In the iron ore industry, balling drums used in pellets production has a long tradition. The main problem areas associated with such drums are therefore well described and to some extent also analysed. In Figure 1, a typical single drum circuit used for pelletizing iron ore is shown.

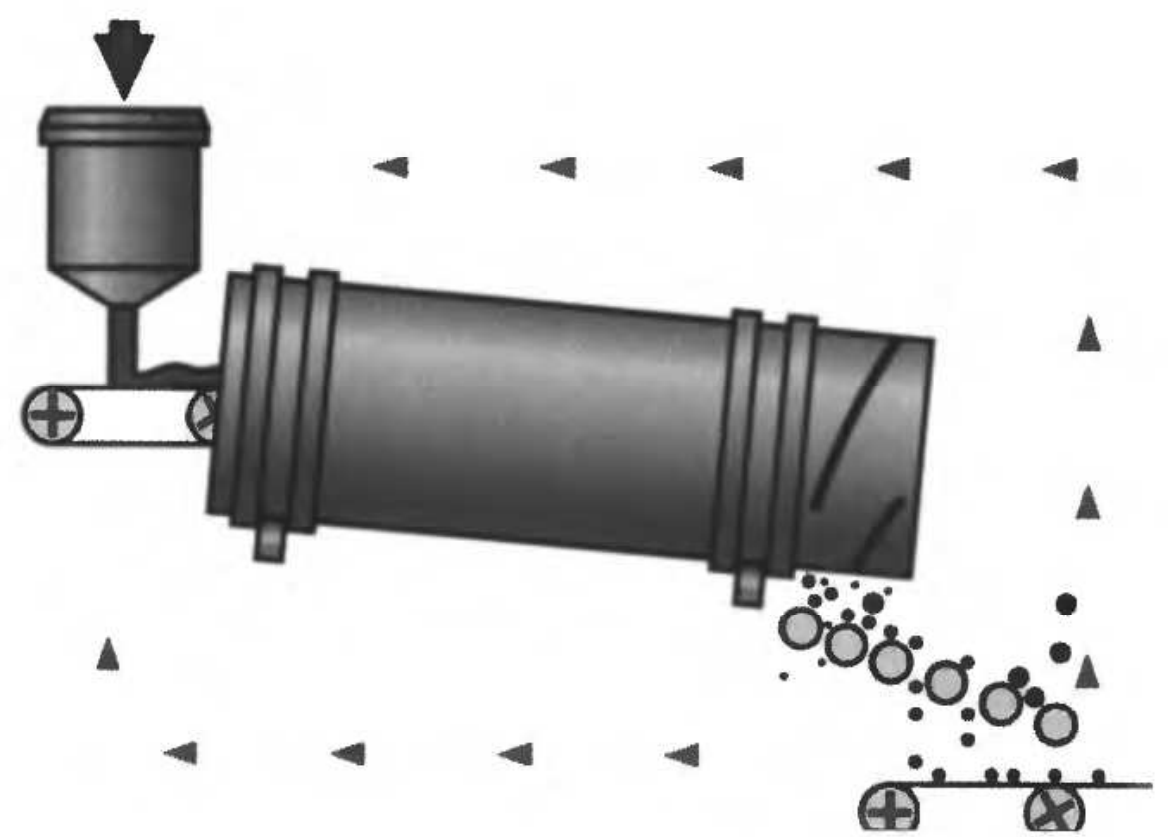

Figure 1. Single balling drum circuit.

\footnotetext{
* Narvik University College, PO Box 385, N-8505 Narvik, Norway, Email: kr@hin.no, Fax: + 4776966810 .

† Narvik University College, PO Box 385, N-8505 Narvik, Norway, Email: pon@hin.no. Fax: + 4776966810 .
} 
The arrows below the drum represents the undersize flow of pellets with too low diameter. These pellets are transported back and fed into the drum together with the fines. The arrows above the drum are the oversize flow of pellets with too big diameter. These pellets are crushed and transported bach to the fines tank. The pellets on the lower right conveyor belt are the onsize pellets, which form the process output.

One problem the iron ore industry has been dealing with for as long as balling drums have been used, is that they tend to give a surging output under some operational conditions. This surging poses a problem for the balling drum circuit only if the amplitudes get too high. A process shutdown will then normally be the result, but danger for some equipment, such as the conveyor belt for recirculation of undersized pellets, is also evident. The major problem with surging lies however, in the subsequent process segment, which is the induration process, also denoted as the 'warm process'. If the input to the warm process is fluctuating, then the efficiency of this segment is reduced, accompanied by a considerably increase in energy consumption and poor product quality. Unfortunately, the conditions which cause the drums output to oscillate, coincidence with those required for good product quality (Cross, 1977; Sastry \& Furstenau 1977). Several attempts have therefore been made to design an automatic control scheme for regulating the amplitude to zero (Wellstead \& Munro, 1976).

So far the problem has only been considered for a single drum and tandem drums even if a pelletizing plant normally consists of several drums (Cross et al., 1977; Wellstead et al., 1978; Sastry, 1993). In this paper we point out why such a strategy may not be optimal and suggest an alternative way to solve the problem.

\section{Preliminary results}

Over the last four decades the problem with surging drums has been investigated from several points of view. A great deal of work has been carried out in developing mathematical models of the balling drum. The first model was established about thirty years ago (Sastry, 1970; Wellstead \& Munro, 1976). This model has infinity dimension, and is as such not useful for control purposes. A lot of knowledge about the system is, however, gained from this model. Later, simplified models has been presented (Cross, 1977). Simulations based on these models reveals that the moisture content in the pellets are the most important process parameter to be controlled if one wishes to stabilize and keep the process stable. The binder, which is added in order to obtain sufficient mechanical strength, tends to have an contrary effect. This suggests that a simple multivariable control could be applied to regulate the moisture content, mechanical strength and the amount of onsized pellets. Unfortunately, this does not turn out to be possible. One of reasons for this is that some plant parameters are hard to measure on line, and consequently difficult to control. An other reason is that small changes in the operating conditions, e.g the moisture content, may cause large changes in the operating point (Cross, 1977). Together with noisy measurements, this offers a big challenge for this approach.

\section{Stabilizing and controlling the drums}

As mentioned in the previous section, a quite simple multivariable control system could be designed if some practical problems were solved. One of the most important 
unsolved problem is to develop a method for on-line measurement of the moisture content in the fines. As far as we are aware of, the best equipment today offer an accuracy no better than $\pm 0.5 \%$ in absolute error (Drügge et al., unpublished), and this is not sufficient, as a change less than this may cause the drum to surge. Alternative ways of stabilizing the drums have been tried, and in the following we describe two different methods described in the literature (Wellstead \& Munro, 1976; Wellstead et al., 1978), and we introduce a new concept as a third method.

\subsection{Method 1}

The simplest method suggested to stabilize the drum circuit is to add moisture in the recycle circuit when the surging occur. If a proper amount of moisture is added then the surging will decrease and finally stop. This method has been implemented on a real drum (Drügge et al., unpublished), but some serious problems were revealed. First of all, a method for detecting that surging really was present was not sufficiently developed. Secondly, as the surging disappear, the system will no longer be observable in the sense that all information needed to decide the amount of moisture to be added in order to keep the process stable without overcompensating, is lost. For this reasons the method was rejected (Drügge et al., unpublished).

Another serious drawback with this method is that it is suboptimal from a energy point of view, as moisture is added without any knowledge about other important parameters, as the amount of binder in the fines. This may result in overcompensating, i.e. too high water content in the onsize pellets, which, in addition to increased energy consumption, will result in poor quality and reduced drum throughput. In other words, the product quality and productivity are not considered in this control scheme.

\subsection{Method 2}

A second method was presented by Wellstead \& Munro (1976). This method, which is the first based on control theoretical analysis, concludes that the surging is a limit cycle caused by to high loop gain. Then the well known method of reducing the gain, which is exactly the same as reducing the amount of recycled undersized pellets, is applied to stabilize the drum. Results from simulations based on the model described in Cross (1977), shows that a reduction of the recycled pellets of about $10-12 \%$ will be sufficient to bring the surging down to an acceptable level. When the drum is stabilized, a multivariable control system for controlling the amount of onsize pellets and the moisture content in the pellets, can be designed. Since the moisture content is an important quality parameter, this model represent therefore a great achievement compared to the previous method.

From an economical or a productivity point of view the method is not optimal, since a mass balance shows that by taking some fraction out of the recycled mass, then the amount of onsize pellets will be reduced accordingly. This method of stabilizing the drum will therefore lower the output of onsize pellets when the input is constant.

As mentioned in the previous section, one of the problems with the first method is that the observability is lost at the same moment as surging disappears. A similar problem appears also in this method. If we assume that the amount of undersized pellets which is removed from the system should be as small as possible, then we should remove just enough to get the system stabilized. If the conditions are then 
changed in such a direction that the system becomes more stable, i.e. the moisture content is increased, then it may be quite difficult to discover that less material could be removed. The problem with unobservability is therefore still present.

\subsection{Method 3, a new approach}

A basic assumption in this method is that the surging does no harm to the pellets quality (Cross, 1977). From a quality point of view there should therefore not be any problem to let the surging be present. The remaining problem then is to make sure that the amount of green pellets transferred to the warm part is constant. In order to obtain this goal, a cluster of drums is controlled collectively, rather than controlling each drum individually. The control scheme may be described in the following way:

1. All drums are operated with surging output, and the amplitude is kept on a desirable value by controlling the water and binder content in the fines.

2. One drum, preferably the one with largest amplitude, is chosen as reference (fixed RPM and return conveyor speed).

3. By adjusting the return conveyors speed the drums phase angel, relative to the reference drum, is controlled in such a way that the amplitude in the total output from all drums is kept at its minimum.

Controlling the amplitude to the desired value will in this setting mean that the quality parameters (the content of moisture and binder) will decide the amplitude. As the amplitudes in practice are not equal, the drum with largest amplitude should preferably be chosen as reference, as this allow us to more easily derive criteria for when the total amplitude is zero. Further more, as this drum normally will have the lowest signal to noise ratio (SNR), it is likely to have the best estimate of amplitude and phase.

The advantages of this method can be summarize as follows:

- The process parameters can be kept at a level which gives high product quality

- The total pelletizing process will consume considerable less energy compared to the situation where the moisture content is kept at a higher lever to avoid surging

- The total capacity (throughput) of the drum is not reduced

- There is no need for an advanced process model as the drums are treated as oscillators with controllable amplitude and frequency

A prerequisite for this method is that the following assumptions hold:

1. it is possible to detect the oscillation and estimate its amplitude, phase and frequency with sufficient accuracy

2. the amplitude is controllable in some range

3. the frequency is controllable in some range

The two latter items are well documented in the literature (Cross, 1977). We will therefore concentrate on the first item in the following section.

\section{Estimation of amplitude, phase and frequency}

\subsection{Signal model}

An extended Kalman filter (EKF) is used for estimation of the frequency, amplitude and phase of the oscillations. The signal model used by the EKF is 


$$
\begin{aligned}
x_{1}(k+1) & =x_{1}(k)+v_{1} \\
x_{2}(k+1) & =x_{1}(k)+x_{2}(k) \\
x_{3}(k+1) & =x_{3}(k)+v_{2} \\
y(k) & =x_{3}(k) \sin x_{2}(k)+z(k)
\end{aligned}
$$

where $x_{1}$ is the phase increment (or frequency), $x_{2}$ is the phase and $x_{3}$ is the amplitude of the oscillation. $\mathbf{v}=\left[v_{1}, v_{2}\right]^{T}$ and $z$ are white, zero mean processes with covariance matrices $\mathbf{Q}=\operatorname{diag}\left(q_{1}, q_{2}\right)$ and $\mathbf{R}$. Locally the state is uniquely determined by the output $y$, but owing to the factor $\sin x_{2}$ in output equation, this does not hold globally. In fact, a simultaneous change of sign in $x_{1}$ and $x_{2}$, or a shift of $x_{2}$ by any number of periods, does not change the output. However, with a reasonable initialization of the EKF this mild nonuniqueness does in general not cause problems. The choice of the matrix $\mathbf{Q}$ is a compromise between accuracy in steady state and capability to track a changing amplitude or frequency. $\mathbf{R}$ is set equal to the covariance of assumed measurement noise of the true, measured output.

\subsection{EKF equations}

Written in a more compact form, equation (1)-(4) are given by

$$
\begin{aligned}
\mathbf{x}_{k+1} & =\mathbf{A} \mathbf{x}_{\boldsymbol{k}}+\mathbf{B} \mathbf{v}_{k} \\
\mathbf{y}_{k} & =g_{k}\left(\mathbf{x}_{k}\right)+z_{k}
\end{aligned}
$$

In the literature two different formulations of the discrete time extended Kalman filter are widely used. The first one is a one-step formulation in terms of the $a$ priori variables, and the second one is a two-step recursion consisting of a time update and a measurement update with a relinearization between the two steps. In this paper the latter formulation is used.

The EKF equations derived from equations (1)-(4) are given by Chui \& Chen (1998):

Time update

$$
\begin{aligned}
& \hat{\mathbf{x}}_{k, k-1}=\mathbf{A} \hat{\mathbf{x}}_{k-1} \\
& \hat{\mathbf{P}}_{k, k-1}=\mathbf{A} \cdot\left[\mathbf{P}_{k-1, k-1}\right] \cdot \mathbf{A}^{T}+\mathbf{B} \cdot \mathbf{Q} \cdot \mathbf{B}^{T}
\end{aligned}
$$

Measurement update

$$
\begin{aligned}
\hat{\mathbf{x}}_{k, k} & =\hat{\mathbf{x}}_{k, k-1}+\mathbf{K}_{k}\left(y_{k}-g_{k}\left(\hat{\mathbf{x}}_{k, k-1}\right)\right) \\
\hat{\mathbf{P}}_{k, k} & =\hat{\mathbf{P}}_{k, k-1}-\mathbf{K}_{k}\left[\frac{\partial g_{k}}{\partial \mathbf{x}_{k}}\left(\hat{\mathbf{x}}_{k, k-1}\right)\right] \hat{\mathbf{P}}_{k, k-1}
\end{aligned}
$$

where

$$
\mathbf{A}=\left[\begin{array}{lll}
1 & 0 & 0 \\
1 & 1 & 0 \\
0 & 0 & 1
\end{array}\right], \quad \mathbf{B}=\left[\begin{array}{ll}
1 & 0 \\
0 & 0 \\
0 & 1
\end{array}\right]
$$


and

$$
\begin{aligned}
\mathbf{G}_{k} & =\left[\frac{\partial g_{k}}{\partial \mathbf{x}_{k}}\right]_{\mathbf{x}=\mathbf{x}_{k, k-1}} \\
& =\left[\begin{array}{lll}
0 & x_{k, k-1}^{(3)} \cos \left(x_{k, k-1}^{(2)}\right) & \sin \left(x_{k, k-1}^{(2)}\right)
\end{array}\right]
\end{aligned}
$$

The filter gain matrix is given by

$$
\mathbf{K}_{k}=\mathbf{P}_{k, k-1}\left[\mathbf{G}_{k}\left(\hat{\mathbf{x}}_{k, k-1}\right)\right]^{T} \cdot\left[\mathbf{G}_{k}\left(\hat{\mathbf{x}}_{k, k-1}\right) \mathbf{P}_{k, k-1} \mathbf{G}_{k}\left(\hat{\mathbf{x}}_{k, k-1}\right)+\mathbf{R}^{T}\right]^{-1}
$$

\subsection{Estimation of the amplitude}

In this work we assume that control of the first harmonic component of the oscillations will give sufficient accuracy for this application. Figure 2 shows a typical situation from a physical plant in LKAB's works in Kiruna, Sweden. The measurement is done with a sampling period of 1 second. As we see, the signal has considerable noise components. The variance is calculated to be approximately $135[\text { tons } / h]^{2}$.

The dark solid-drawn line is the estimate from the Kalman filter, and the light solid-drawn line is the real, noisy signal. The filter model is based on a sinusoid, which in this application corresponds to the first harmonic of the oscillation. The solid-drawn line is the filters estimate of the amplitude. An important question at this stage is as follows: what is the lowest possible amplitude the filter is capable of

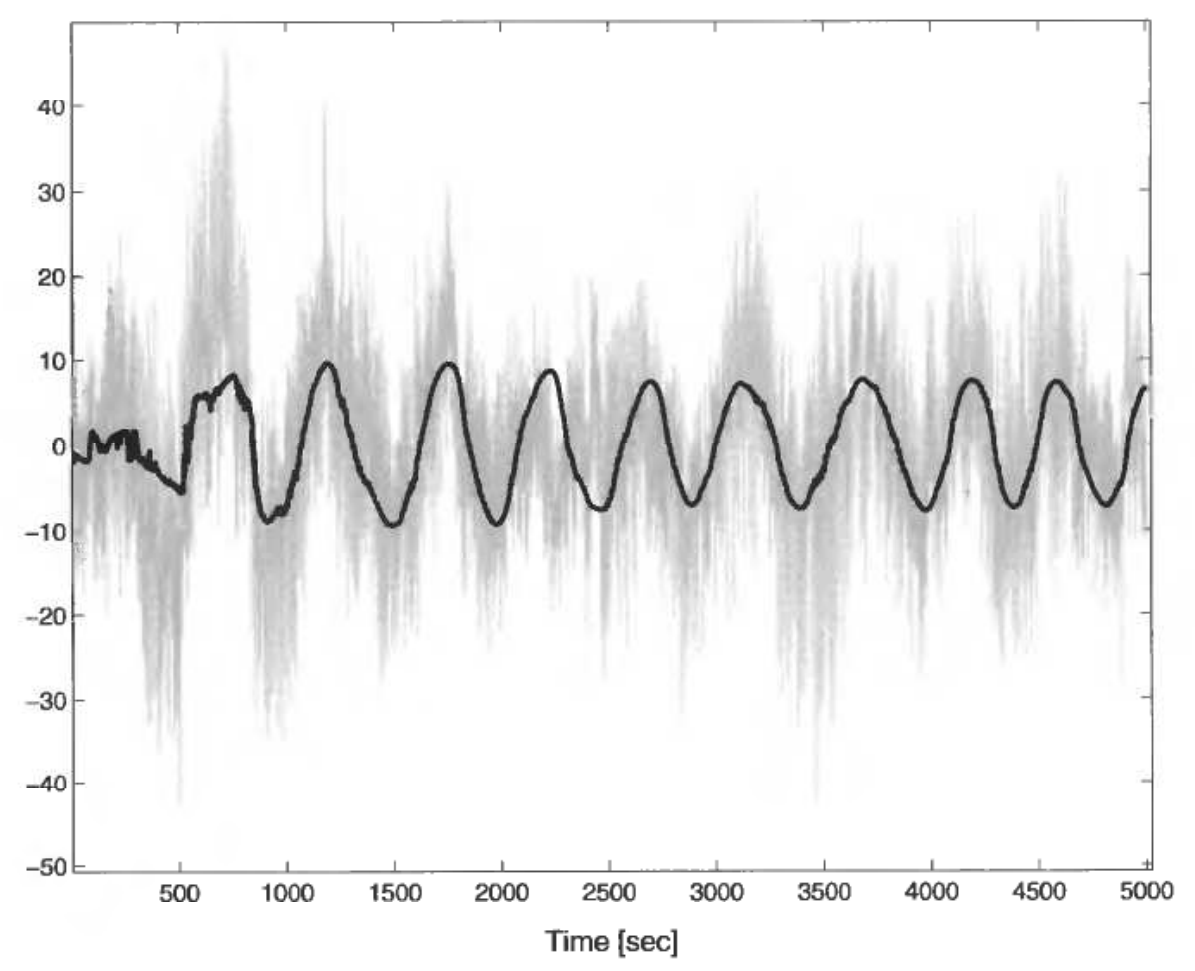

Figure 2. Variations around the operating point. 


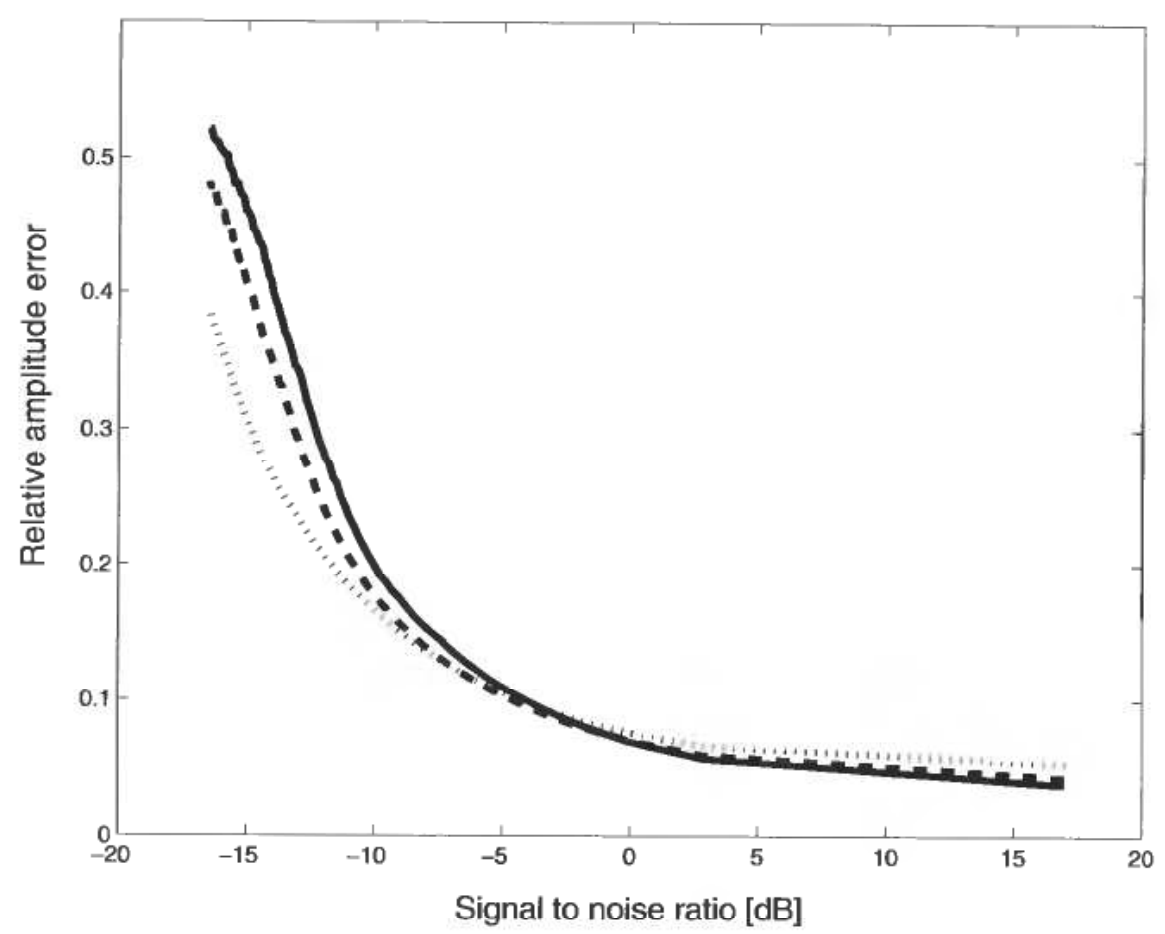

Figure 3. Relative amplitude error vs. signal-to-noise ratio.

detecting, given a specified level of noise. If we permit the amplitude to get below this limit we will no longer be able to control the phase angel to the desired value.

The signal to noise ratio is given by the following equation

$$
S N R=10 \log \frac{A^{2}}{2 \operatorname{Var}(v)}
$$

where $A$ is the amplitude of the oscillation and $v$ is the signal noise, which is assumed to be white.

In Figure 3 the filters signal to noise ratio curve with respect to relative amplitude error is shown.

In Figure 3, the solid line is for $A=20($ ton $/ h)$, the dashed line in the middle represents $A=10($ ton/h $)$, and the dotted line represents $A=5($ ton/h). Clearly the area where the thresholding phenomenon occurs depends on the amplitude.

\subsection{Estimation of the phase}

In Figure 4 the filters signal to noise ratio curve with respect to phase error is shown,

As in subsection 4.2, the thresholding phenomenon occurs at different SNR levels for different amplitudes. It is also clear from Figure 4 that the filters phase estimate is more robust to noise that the amplitude estimate.

\subsection{Estimation of the frequency}

In Figure 5 the filters signal to noise ratio curve with respect to frequency error is shown. 


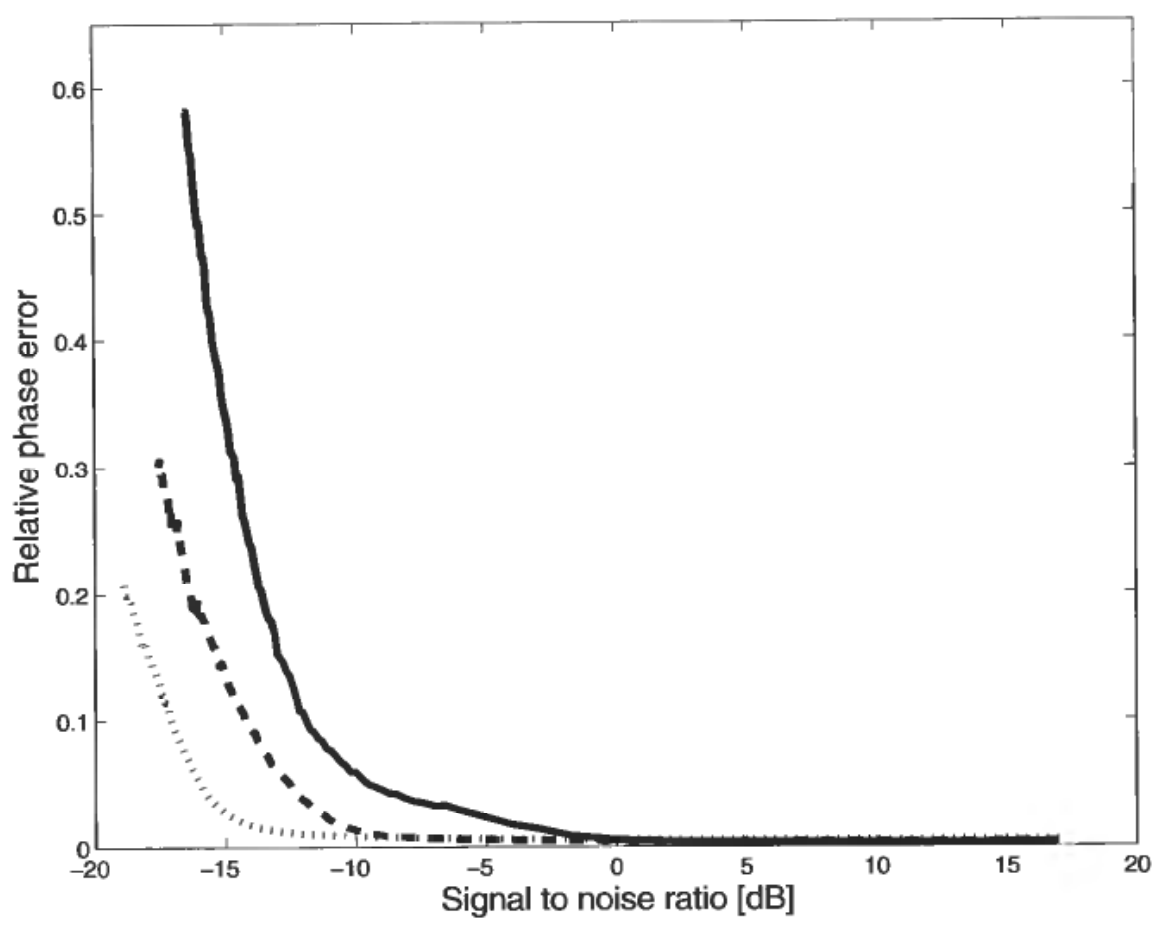

Figure 4. Phase error vs. signal-to-noise ratio.

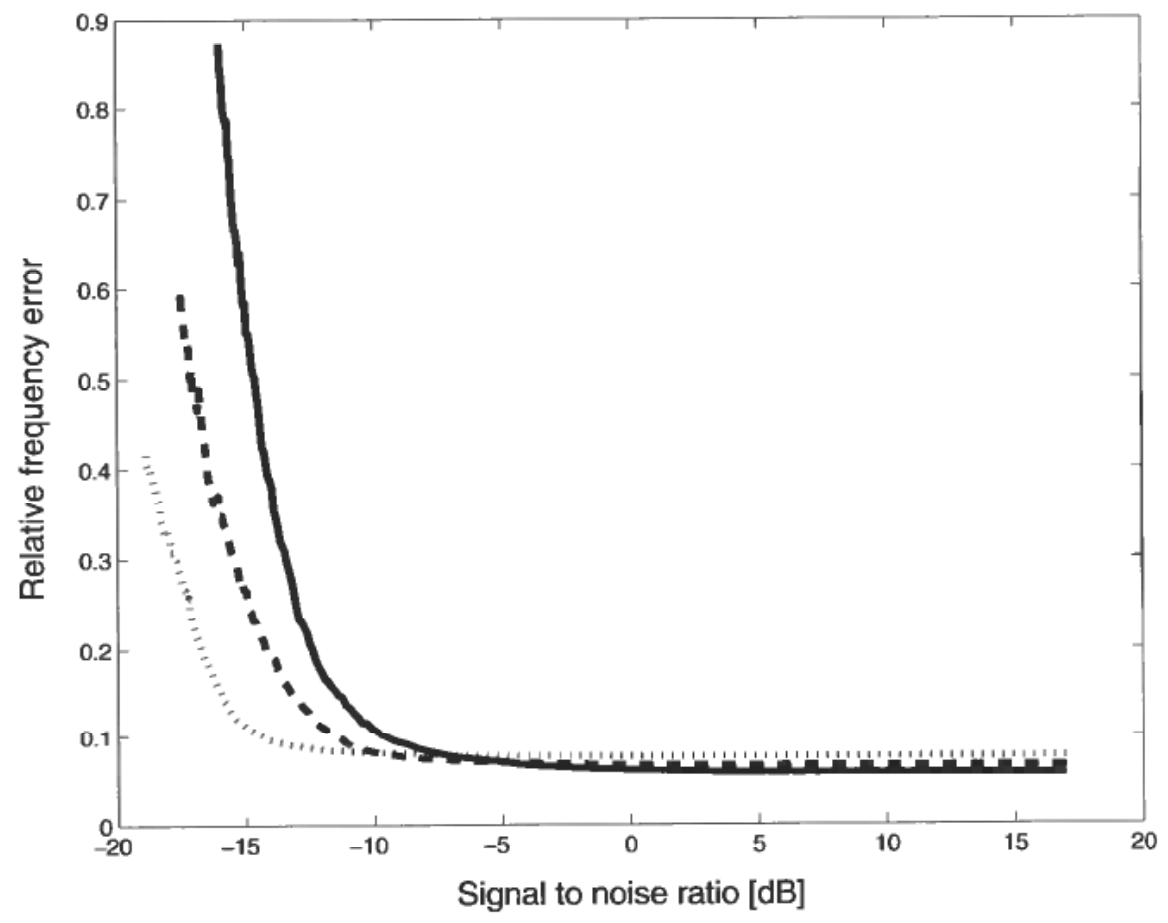

Figure 5. Frequency error vs. signal-to-noise ratio. 

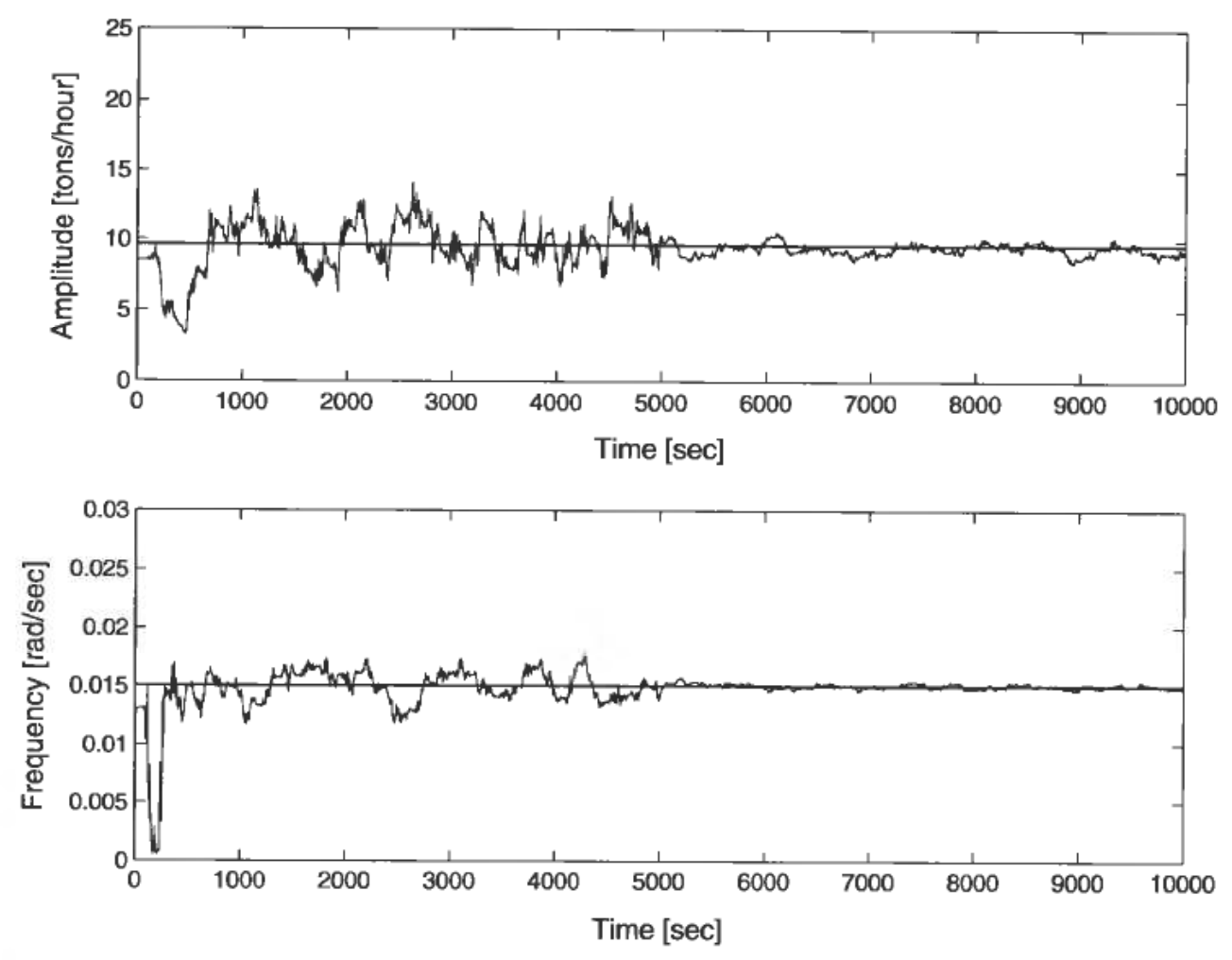

Figure 6. Amplitude and frequency estimate.

Also in this case we see the same pattern as in the previous two subsections. It is also clearly seen from the figure that the frequency estimate is more robust to noise than the amplitude estimate.

\subsection{Discussion}

From the previous subsections, we see that it is the filters ability to estimate the amplitude which is the most critical part. Using the case with $A=10[$ tons $/ h]$ as example, we see that thresholding in the amplitude estimate will occur for SNR below approximately $-10 \mathrm{~dB}$. For the frequency and phase estimate thresholding will occur below $-13 \mathrm{~dB}$ and $-12 \mathrm{~dB}$ respectively.

In a typical plant the noise level will normally be between $50[\text { tons } / h]^{2}$ and 250 $[t o n s / h]^{2}$. Within these limits we may expect that the filter will be able to track a signal with a amplitude down to $7.5[$ tons $/ h]$, i.e SNR $=-10 \mathrm{~dB}$. Repeated simulations shows that the filter in fact does work in this situation. We should, however, not expect the filter to have good performance close to the thresholding point. In Figure 6 a representative result is shown. In the interval $0 \leqslant t \leqslant 5000$ the signal to noise ratio is $-9.8 \mathrm{~dB}$ and in the interval $5000 \leqslant t \leqslant 10000$ it is increased to $3 \mathrm{~dB}$ by decreasing the signal measurement noise.

\section{Results from simulations}

In this section results from simulations are shown.

A simple model based on Van der Pol's equation is used to describe the drums 

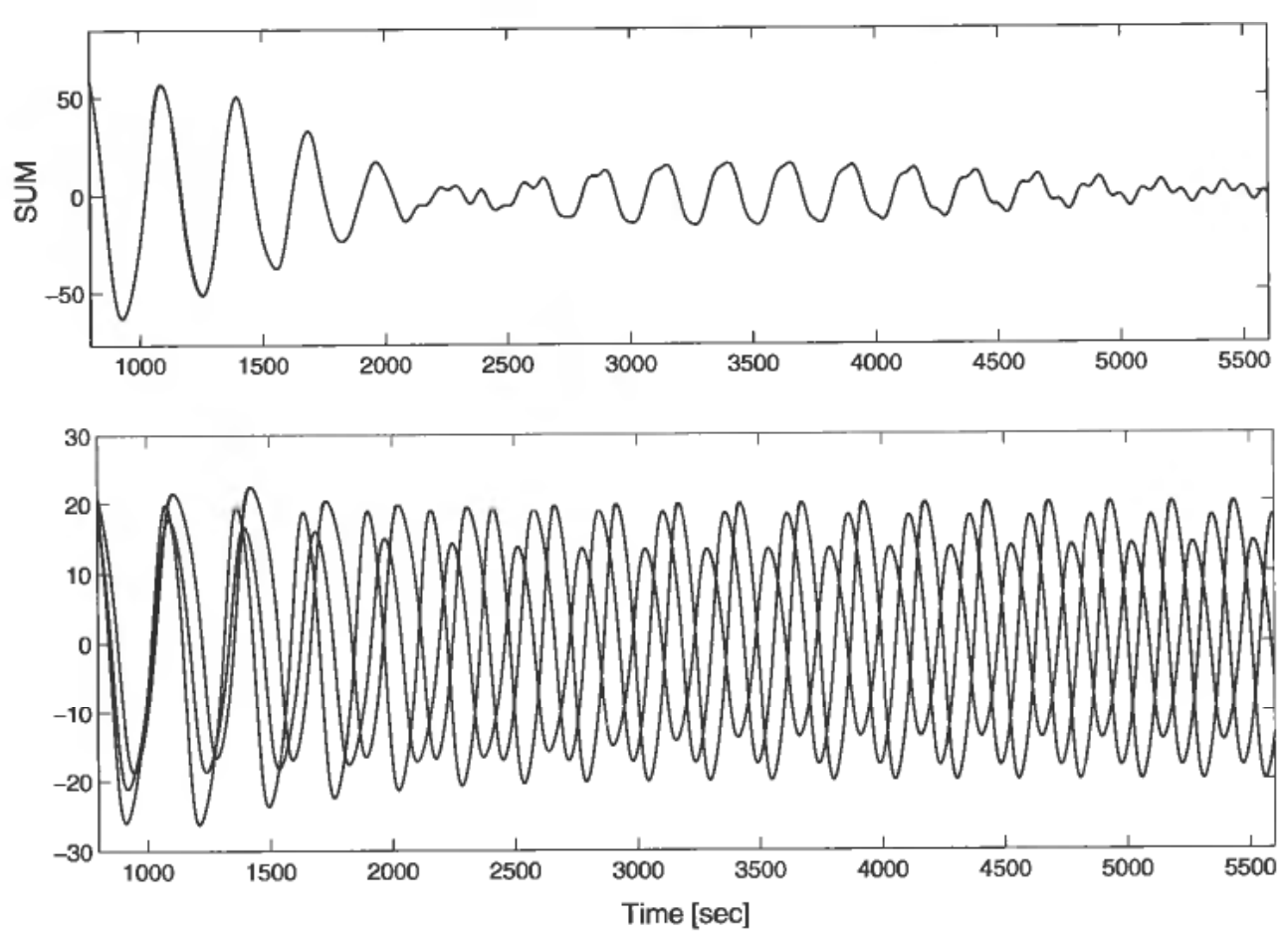

Figure 7. Result from simulation with a cluster of three balling drums.

oscillating behavior. This model may only be used for a quite narrow range of the process parameters, as it does not adequately describe the transition between the drums uscillation mode and steady mode. It should be pointed out that this model gives no information about the plants efficiency, it is only intended to illustrate the proposed control scheme.

A standing assumption in this section is that the moisture content in the pellets is sufficiently low, so that the drums will oscillate. It is also assumed that the system is deterministic.

The simulated system is a cluster of three drums. The systems outputs are each drums onsize flow and the total flow. One drum is chosen as phase reference drum, and PID controllers are applied for controlling each of the slave-drums phase angel relative to the reference drum. In Figure 7, the upper figure shows the total flow and the lower figure shows each drums onsize flow. As in Figure 2, only the variation around the operating point is shown.

In the first $800 \mathrm{sec}$. the drums are started and the oscillations are established. After this time delay the PID controllers starts to adjust the phase angels, and the amplitude in the total flow starts to decrease. From Figure 7 we clearly see that the control is quite slow. Experiments with this model shows that it is quite difficult to speed up the control. The reason for this is somewhat unclear, however we expect that one of the following modifications may allow faster control:

(a) Use of a multivariable controller structure rather than single loop controllers

(b) The basis for the signal model used in the EKF has no local support, so that any changes will affect the output for all future. A change to a basis with local support e.g. a wavelet basis may therefore be desirable. 


\section{Concluding remarks}

In this paper we have suggested a new approach to the old problem of controlling the balling drums used for pelletizing iron ore. A prerequisite for this method is that is has to be possible to estimate the oscillations amplitude, phase and frequency with sufficient accuracy. We have investigated the possibility for using an extended Kalman filter for this purpose. In section 4 we show that this is indeed possible.

The proposed control scheme has been simulated for a plant consisting of three drums. One major problem which remains to be solved is that it takes too long time for the output to settle down. One of the reasons for this may be that the basis for the signal model is a trigonometric basis, which has no local support. A possible solution of this problem may therefore be to use a signal model with local support, e.g. a wavelet basis. Another possible solution may be to use a multivariable controller structure rather than single loop controllers, which are used in the simulation presented in section 5 .

\section{Acknowledgement}

The authors are grateful to LKAB, Sweden, for support both financially and practical during this research. In particular Mr. Magnus Rutfors deserves a great thanks for providing us with measurement data from the real process.

\section{References}

ChU,, C. K. \& CHEN, G. (1998). Kalman Filtering with Real-time Applications, Springer Verlag.

Cross, M. (1977). Mathematical model of balling-drum circuit of a pelletizing plant, Ironmaking and steelmaking No. 3.

Cross, M., Young, R. W., Wellstead, P. E. \& Gibson, R. D. (1977). The mathematical modelling and control aspects of the pelletizing of iron ore. Proceedings of the 2nd international symposium of agglomeration, AIME, New York.

DrÜGGE, R., ERIKSON., O. \& RutFors, M. Unpublished work, LKAB Sweden.

SASTRY, K. V. S. (1970). The agglomeration of particulate materials by green pelletization, $\mathrm{Ph} . \mathrm{D}$ thesis.

SASTRY, K. V. S. \& Furstenau, D. W. (1977). Kinetic and process analysis of the agglomeration of particulate materials by green pelletization. Proccedings of the 2nd international symposium of agglomeration, AIME.

Sastry, K. V. S. (1993). Process engineering of agglomeration systems. Proceedings of the sixth international symposium of agglomeration.

Wellstead, P. E. \& MunRo, N. (1976). Multivariable control of cold iron ore agglomeration plant.

Wellstead, P. E., Cross, M., Munro, N. \& Ibrahim, D. (1978). On the design and assessment of control schemes for balling-drum circuits used in pelletizing, International Journal of Mining Processing, 5, 45-67 